\title{
Relative generalized Hamming weights and extended weight polynomials of almost affine codes
}

\author{
Trygve Johnsen and Hugues Verdure \\ UiT -The Arctic University of Norway, N-9037 Troms $\varnothing$, Norway \\ Trygve. Johnsen@uit.no Hugues. Verdure@uit. no
}

\begin{abstract}
This paper is devoted to giving a generalization from linear codes to the larger class of almost affine codes of two different results. One such result is how one can express the relative generalized Hamming weights of a pair of codes in terms of intersection properties between the smallest of these codes and subcodes of the largest code. The other result tells how one can find the extended weight polynomials, expressing the number of codewords of each possible weight, for each code in an infinite hierarchy of extensions of a code over a given alphabet. Our tools will be demi-matroids and matroids.
\end{abstract}

Keywords: Pairs of almost affine codes, relative generalized Hamming weights, extended weight polynomials

\section{Introduction}

We will focus on almost affine codes as defined in [14], that is: $C \subset F^{n}$ for some finite alphabet $F$, and the projection $C_{X}$ has cardinality $|F|^{s}$ for a non-negative integer $s$ for each $X \subset\{1, \cdots, n\}$. It is well known that this is a class of codes, which contain linear codes over fields $F$ as a proper subclass. The intermediate class of affine codes are translates of linear codes. Another intermediate class is that of multilinear codes. It is also well known ([14]) that $C$ defines a matroid $M_{C}$ through the rank function

$$
r(X)=\log _{|F|}\left|C_{X}\right| .
$$

Such codes were studied in connection with access structures over $E=\{1,2, \cdots, n\}$ and are strongly related to ideal perfect secret sharing schemes for such access structures. See e.g. [14].

In this note we will demonstrate how two different results for linear codes can be generalized to find analogous results for almost affine codes in general.

First, we will recall some known results and terminology for almost affine codes in Section 2. Then, in Section 3, we will study generalized Hamming weight (RLDP, in the sense of [2]) of pairs $C_{2} \subset C_{1}$ of almost affine codes, and we will investigate to which extent it is possible to generalize the results in [12] and [15], 
where one only treats linear codes. There one expresses these relative generalized weights as the minimum weights of subcodes of $C_{1}$ of various dimensions, intersecting $C_{2}$ only in the zero element. In one of our two main results, Theorem 2, we show an analogue of this result for almost affine codes. In Remark 3 we show, however, that the situation is not completely like in the case of linear codes.

There are many applications of relative generalized Hamming weights, as referred to in [12] and [15], for pairs of linear codes. In addition, relating to secret sharing schemes, which is a particularly natural topic, when working with almost affine codes, we would like to mention the significance of relative generalized Hamming weights described in [11] and [3].

In the last chapter, Section 4, we study another aspect of the relationship between almost affine codes and matroids. In [6], and in [5, p. 323], one points out that for linear block codes of length $n$ over a finite field $\mathbb{F}_{q}$, one can produce an infinite series of codes by extending the alphabet to $\mathbb{F}_{q^{s}}$, for $s=1,2, \cdots$, and nevertheless find polynomials $A_{0}, \cdots, A_{n}$, such that $A_{j}\left(q^{s}\right)$ computes the number of codewords of weight $j$, for all $s$ simultaneously, for each of $j=0, \cdots, n$. We will show that a corresponding result holds for almost affine codes, and we use the arguments in $[7$, Section 3] as a stepping stone to find weight polynomials for a similar infinite series of almost affine codes $C^{s}$, all of the same block length, but over growing alphabets $F^{s}$ as $s$ grows. A main point in the linear case is that the polynomials $A_{j}$ are only dependent on the associated matroid of $C$, and that we have matroids that play a completely analogous role, and that are equally simple to handle in the general case of almost affine codes.

\section{Matroids, demi-matroids and almost affine codes}

In this section, we essentially recall relevant material that will be needed in the sequel, and we do not claim to have any new results here. We refer to [13] for the theory of matroids, to [1] for an introduction on demi-matroids and to [14] for an introduction on almost affine codes, and we will use their notation.

\subsection{Matroids and demi-matroids}

A matroid is a combinatorial structure that extend the notion of linear (in)dependency. There are many equivalent definitions, but we will give just one here.

Definition 1. A matroid is a pair $M=(E, r)$ where $E$ is a finite set, and $r$ a function on the power set of $E$ into $\mathbb{N}$ satisfying the following axioms:

(R1) $r(\emptyset)=0$,

(R2) for every subset $X \subset E$ and $x \in E, r(X) \leqslant r(X \cup\{x\}) \leqslant r(X)+1$,

(R3) for every $X \subset E$ and $x, y \in E$, if $r(X)=r(X \cup\{x\})=r(X \cup\{y\})$, then $r(X \cup\{x, y\})=r(X)$.

Demi-matroids were introduced in [1]. They are a generalization of matroids in the following way: 
Definition 2. A demi-matroid is a pair $M=(E, r)$ where $E$ is a finite set, and $r$ a function on the power set of $E$ into $\mathbb{N}$ satisfying axioms (R1) and (R2) above. The rank of $M$ is $r(E)$.

Matroids and demi-matroids have duals defined in the following way:

Proposition 1. Let $M=(E, r)$ be a matroid (respectively a demi-matroid). Then $M^{*}=\left(E, r^{*}\right)$ with $r^{*}$ defined as

$$
r^{*}(X)=|X|+r(E \backslash X)-r(E)
$$

is a matroid (respectively a demi-matroid). Moreover, $\left(M^{*}\right)^{*}=M$.

The matroid (respectively demi-matroid) $M^{*}$ is called the dual (respectively the dual or first dual) of $M$. It has rank $|E|-r(M)$. Demi-matroids have another dual, called the supplement dual or second dual. See [1, Theorem 4]:

Proposition 2. Let $M=(E, r)$ be a demi-matroid. Then $\bar{M}=(E, \bar{r})$ with $\bar{r}$ defined as

$$
\bar{r}(X)=r(E)-r(E \backslash X)
$$

is a demi-matroid. Moreover, we have $\overline{\bar{M}}=M$ and $\overline{M^{*}}=\bar{M}^{*}$.

\subsection{Almost affine codes}

Almost affine codes were first introduced in [14], and are a combinatorial generalization of affine codes.

Definition 3. An almost affine code over a finite alphabet $F$, of length $n$ and dimension $k$, is a subset $C \subset F^{n}$ such that $|C|=|F|^{k}$ and such that for every subset $X \subset E=\{1, \cdots, n\}$,

$$
\log _{|F|}\left|C_{X}\right| \in \mathbb{N}
$$

where $C_{X}$ is the puncturing of $C$ with respect to $E \backslash X$.

An almost affine subcode of $C$ is a subset $D \subset C$ which is itself an almost affine code over the same alphabet.

Remark 1. Any linear or affine code is obviously an almost affine code.

To any almost affine code $C$ of length $n$ and dimension $k$ on the alphabet $F$, we can associate a matroid $M_{C}$ on the ground set $E=\{1, \cdots, n\}$ and with rank function

$$
r(X)=\log _{|F|}\left|C_{X}\right|,
$$

for $X \subset E$. 
Definition 4. Let $C$ be a block code of length n, and let $\boldsymbol{c} \in C$ be fixed. The $\boldsymbol{c}$-support of any codeword $\boldsymbol{w}$ is

$$
\operatorname{Supp}(\boldsymbol{w}, \boldsymbol{c})=\left\{i, \boldsymbol{c}_{i} \neq \boldsymbol{w}_{i}\right\}
$$

The c-support of $C$ is

$$
\operatorname{Supp}(C, \boldsymbol{c})=\bigcup_{\boldsymbol{w} \in C} \operatorname{Supp}(\boldsymbol{w}, \boldsymbol{c}) .
$$

Note that the $\boldsymbol{c}$-support of an almost affine code is independent of the choice of $\boldsymbol{c} \in C$ (see [8, Lemma 1]), and it will therefore be denoted by $S u p p(C)$ without reference to any codeword. This observation gives rise to:

Definition 5. The weight of an almost code $C$ is $w(C)=|\operatorname{Supp}(C)|$.

Definition 6. Let $C$ be an almost affine code of length $n$, and let $\boldsymbol{c} \in F^{n}$ be fixed. Then

$$
C(X, \boldsymbol{c})=\left\{\boldsymbol{w} \in C, \boldsymbol{w}_{X}=\boldsymbol{c}_{X}\right\},
$$

where $\boldsymbol{c}_{X}$ is the projection of $\boldsymbol{w}$ to $X$. Such a subcode of $C$ is called a standard subcode.

This might be empty, or not be an almost affine code, but when we take $c \in C$, we get the following ([14, Corollary 1$])$ :

Proposition 3. Let $C$ be an almost affine code of length $n$ and dimension $k$ over the alphabet $F$. Let $\boldsymbol{c} \in C$. Let $X \subset\{1, \cdots, n\}$. Then $C(X, \boldsymbol{c})$ is an almost affine subcode of $C$. Its asscociated matroid $M_{C(X, c)}$ is the contracted matroid $M_{C} / X$ with rank function $\rho$ given by

$$
\rho(Y)=r(X \cup Y)-r(X)
$$

where $r$ is the rank function of the matroid $M_{C}$. In particular,

$$
|C(X, \boldsymbol{c})|=|F|^{k-r(X)} .
$$

Remark 2. Not all subcodes of $C$ are of the form $C(X, \boldsymbol{c})$, i.e. not all subcodes are standard subcodes.

Corollary 1. Every almost affine code $C$ of dimension $k$ has almost affine subcodes of dimension $0 \leqslant i \leqslant k$.

\subsection{Generalized Hamming weights}

For a demi-matroid $D=(E, r)$ of rank $n-k$ we define:

Definition 7. The generalized Hamming weights for a demi-matroid of dimension $k$ are

$$
m_{i}(D)=\min \{|X|, n(X)=|X|-r(X)=i\}
$$

for $1 \leqslant i \leqslant k$. 
Definition 8. The generalized Hamming weights for an almost affine code $C$ of dimension $k$ are

$$
d_{i}(C)=m_{i}\left(M_{C}^{*}\right)=\min \left\{|X|,|X|-r^{*}(X)=\bar{r}(X)=i\right\}
$$

for $1 \leqslant i \leqslant k$, where $r^{*}$ is the rank function of $M_{C}^{*}$, and $\bar{r}$ is the rank function of $\overline{M_{C}}$.

In fact the following was proved in [9]:

Theorem 1. Let $C$ be an almost affine code of length $n$ and dimension $k$ on an alphabet $F$ of cardinality $q$, and let $\boldsymbol{c} \in C$. Then the generalized Hamming weights for $C$ are

$$
\begin{gathered}
d_{i}(C)=\min \{|\operatorname{Supp}(D)|, D \text { is an almost affine subcode of dim. } i \text { of } C\}= \\
\min \{|\operatorname{Supp}(D)|, D \text { is a standard subcode of dim. } i \text { of } C\}= \\
n-\max \left\{|X|,|C(X, \boldsymbol{c})|=q^{j}\right\},
\end{gathered}
$$

for $1 \leqslant i \leqslant k$.

\section{Equivalent formulations of some Hamming weights of pairs of codes}

From [10] we have:

Proposition 4. Let $C_{2} \subset C_{1}$ be two almost affine codes with rank functions $r_{2}$ and $r_{1}$. Then the pair $(E, \rho)$ is a demi-matroid, for $\rho=r_{1}-r_{2}$.

Definition 9. For $0 \leqslant i \leqslant \operatorname{dim} C_{1}-\operatorname{dim} C_{2}$, we define the $R L D P$ (Relative Length/Dimension Profile), or relative generalized Hamming weight, of the pair $\left(C_{1}, C_{2}\right)$ as follows:

$$
m_{i}=\min \{|X|, \bar{\rho}(X)=i\} .
$$

We observe that if $C_{2}=0$, this is the $d_{i}$ associated to $C_{1}$.

For linear codes the most usual way in the literature (see e.g. [12]) to express the $m_{i}$ is perhaps as

$$
\min \left\{|X| \mid \operatorname{dim}\left(C_{1}(E \backslash X, \underline{0})-\operatorname{dim}\left(C_{2}(E \backslash X, \underline{0}) \geqslant i\right\} .\right.\right.
$$

It is easy to see that our Definition 9 above of the $m_{i}$ gives the same values for linear codes. In [12, Lemma 1], and [15, Prop. 2], however, one gives an alternative, and less trivial reformulation of the $m_{i}$ for linear codes. This is as:

$$
\min \left\{w(D),\left|D \cap C_{2}\right|=1, D \subset C_{1} \text { is a linear subcode with } \operatorname{dim} D=i\right\} .
$$

Another, similar variant is given in [15, Prop.4]. We will now investigate the possibility of a reformulation of the $m_{i}$ in analogous ways, not only for linear codes, but for almost affine codes in full generality. Our result is given in the theorem below: 
Theorem 2. Let $C_{2} \subset C_{1}$ be a pair of almost affine codes with associated demimatroid $(E, \rho)$. Then for $0 \leqslant i \leqslant \operatorname{dim} C_{1}-\operatorname{dim} C_{2}$,

$m_{i}=\min \left\{w(D),\left|D \cap C_{2}\right|=1, D \subset C_{1}\right.$ is a standard subcode with $\left.\operatorname{dim} D=i\right\}$.

Proof. Let $b_{i}$ be the right hand side of the above equality. We fix $\boldsymbol{v} \in C_{2}$. All the standard subcodes, and all supports, considered in this proof, are with respect to $\boldsymbol{v}$, and we omit its reference in the rest of the proof. For simplicity, denote $k_{j}=\operatorname{dim} C_{j}$ and $r_{j}=r_{C_{j}}$ for $j=1,2$.

Let $X \subset E$ be such that $|X|=m_{i}$ and $\bar{\rho}(X)=i$, that is

$$
k_{1}-r_{1}(E \backslash X)-k_{2}+r_{2}(E \backslash X)=i
$$

or equivalently

$$
\operatorname{dim} C_{1}(E \backslash X)-\operatorname{dim} C_{2}(E \backslash X)=i .
$$

We have the obvious inclusions

$$
\operatorname{Supp}\left(C_{2}(E \backslash X)\right) \subset \operatorname{Supp}\left(C_{1}(E \backslash X)\right) \subset X .
$$

We claim that the second inclusion is actually an equality. Indeed, if not, let $y \in X \backslash \operatorname{Supp}\left(C_{1}(E \backslash X)\right)$ and consider $Y=X \backslash\{y\}$. Then, for $j=1,2$, if $\boldsymbol{w} \in$ $C_{j}(E \backslash X), \boldsymbol{w}_{y}=\boldsymbol{v}_{y}$ since $y$ is not in the support, and in turn, the natural inclusions

$$
C_{j}(E \backslash Y) \subset C_{j}(E \backslash X)
$$

are equalities. This contradicts the minimality of $X$ since $Y$ also satisfies $\bar{\rho}(Y)=$ $\bar{\rho}(X)=i$.

Let $Z \subset E \backslash X$ be a maximal independent subset of $E \backslash X$ for the matroid $M_{C_{2}}$, that is

$$
|Z|=r_{2}(Z)=r_{2}(E \backslash X) .
$$

Let $Z^{\prime} \subset X$ be such that $Z \cup Z^{\prime}$ is a basis of $M_{C_{2}}$. Obviously, we have

$$
r_{2}\left(Z^{\prime}\right)=\left|Z^{\prime}\right|=k_{2}-|Z|=k_{2}-r_{2}(E \backslash X) .
$$

Let $W=X-Z$. Note that $Z \cup Z^{\prime} \subset E \backslash W$. Then

$$
C_{2} \cap C_{1}(E \backslash W)=\{\boldsymbol{v}\} .
$$

Namely,

$$
\boldsymbol{v} \in C_{2} \cap C_{1}(E \backslash W)=C_{2}(E \backslash W)
$$

and

$$
\operatorname{dim} C_{2}(E \backslash W)=k_{2}-r_{2}(E \backslash W)=0 .
$$

Moreover, we have

$$
\begin{aligned}
r_{1}(E \backslash W) & \geqslant r_{1}(E \backslash X)+\left|Z^{\prime}\right| \\
& \leqslant k_{1}-k_{2}+r_{2}(E \backslash X)-i+k_{2}-r_{2}(E \backslash X) \\
& \leqslant k_{1}-i
\end{aligned}
$$


that is,

$$
\operatorname{dim} C_{1}(E \backslash W) \geqslant i .
$$

Take now any standard subcode of $C_{1}(E \backslash W)$ of dimension $i$. Then of course we have

$$
\boldsymbol{v} \in D \cap C_{2} \subset C_{1}(E \backslash W) \cap C_{2}=\{\boldsymbol{v}\}
$$

and

$$
\operatorname{Supp}(D) \subset \operatorname{Supp}\left(C_{1}(E \backslash W)\right) \subset \operatorname{Supp}\left(C_{1}(E \backslash X)\right)=X,
$$

which implies that

$$
b_{i} \leqslant \operatorname{Supp}(D) \leqslant|X|=m_{i} .
$$

For the converse, let $Y \subset E$ be such that $\left|C_{1}(Y) \cap C_{2}\right|=1$. Then $C_{1}(Y) \cap C_{2}=$ $\{\boldsymbol{v}\}$. Assume that $w\left(C_{1}(Y)\right)=b_{i}$ and $\operatorname{dim} C_{1}(Y)=i$. Let $Y^{\prime}=E \backslash \operatorname{Supp}\left(C_{1}(Y)\right)$. Obviously, $Y \subset Y^{\prime}$. Let $\boldsymbol{w} \in C_{1}(Y)$. For any $y \in Y^{\prime}, y \notin \operatorname{Supp}\left(C_{1}(Y)\right)$ so that $\boldsymbol{w}_{y}=\boldsymbol{v}_{y}$, and in turn $\boldsymbol{w} \in C_{1}\left(Y^{\prime}\right)$. Hence, the natural inclusion $C_{1}\left(Y^{\prime}\right) \subset C_{1}(Y)$ is actually an equality.

Let $X=E-Y^{\prime}$. Then we have

$$
|X|=\left|E \backslash Y^{\prime}\right|=\mid E \backslash\left(E \backslash \operatorname{Supp}\left(C_{1}(Y)\right)|=| \operatorname{Supp}\left(C_{1}(Y)\right) \mid=b_{i}\right.
$$

and

$$
C_{2}(E \backslash X)=C_{2}\left(Y^{\prime}\right)=C_{2} \cap C_{1}\left(Y^{\prime}\right)=C_{2} \cap C_{1}(Y)=\{\boldsymbol{v}\},
$$

which implies that

$$
\operatorname{dim} C_{1}(E \backslash X)-\operatorname{dim} C_{2}(E \backslash X)=\operatorname{dim} C_{1}\left(Y^{\prime}\right)-0=\operatorname{dim} C_{1}(Y)=i
$$

and finally

$$
m_{i} \leqslant|X|=b_{i}
$$

\subsection{An open question concerning subcodes}

Remark 3. Let

$$
b_{i}^{\prime}=\min \left\{w(D),\left|D \cap C_{2}\right|=1, D \subset C_{1} \text { is a subcode with } \operatorname{dim} D=i\right\},
$$

that is we allow $D$ to be any subcode, not only a standard subcode. Obviously, for $1 \leqslant i \leqslant k_{1}-k_{2}$, we have

$$
m_{i}=b_{i} \geqslant b_{i}^{\prime} .
$$

It is an open question whether the last inequality is an equality, and will be the topic of further research. For linear codes, [15, Proposition 2] gives an analogous statement with equality. On the other hand, while $b_{i}$ is defined just for $0 \leqslant i \leqslant$ $k_{1}-k_{2}$ (for $i>k_{1}-k_{2}$ it is not difficult to show that any standard subcode of $C_{1}$ will have a non-trivial intersection with $\left.C_{2}\right), b_{i}^{\prime}$ might be defined for $i>k_{1}-k_{2}$. Consider namely the following codes: let $F=\{0,1,2,3\}$ and $C_{1}=F^{3}$. Let $C_{2}$ and $D$ be the subcodes

$\{000,012,023,031,103,110,121,132,201,213,222,230,302,311,320,333\}$ 
and

$\{000,011,022,033,102,113,120,131,203,210,221,232,301,312,323,330\}$

respectively. Both subcodes have dimension 2 , while $C_{1}$ has dimension 3 . But we have $C_{2} \cap D=\{000\}$ and $\operatorname{dim} D>\operatorname{dim} C_{1}-\operatorname{dim} C_{2}=1$. Hence $b_{2}^{\prime}$ is defined (and is at most 3 ), while $m_{2}$ and $b_{2}$ could be said to be $\infty$ if one insists on defining them.

\section{Extended weight polynomials of almost affine codes}

In [6], and in [5, p. 323], one points out that for linear block codes of length $n$ over a finite field $\mathbb{F}_{q}$, one can produce an infinite series of codes by extending the alphabet to $\mathbb{F}_{q^{s}}$, for $s=1,2, \cdots$, and nevertheless find polynomials $A_{0}, \cdots, A_{n}$, such that $A_{j}\left(q^{s}\right)$ computes the number of codewords of weight $j$, for all $s$ simultaneously, for each of $j=0, \cdots, n$. Hence knowledge of a finite number of coefficients of the $A_{j}$ compute an infinite number of weights. We will show that a corresponding result holds for almost affine codes, and we will mimick the arguments in [7, Section 3] to find weight polynomials for an infinite series of almost affine codes $C_{s}$, which we will now define.

Let $q=|F|$, where $F$ is the alphabet over which an almost affine code $C$ of block length $n$ is defined. Then $C^{s}$ is a code of block length $n$ over the alphabet $F^{s}$, if an element $\left(\left(c_{1,1}, \cdots, c_{1, n}\right), \cdots,\left(c_{s, 1}, \cdots, c_{s, n}\right)\right)$ instead is interpreted as:

$$
\left(\left(c_{1,1}, \cdots, c_{s, 1}\right), \cdots,\left(c_{1, n}, \cdots, c_{s, n}\right)\right) .
$$

It is then automatic that $\left|\left(C^{s}\right)_{X}\right|=\left(q^{s}\right)^{r}$ if $\left|C_{X}\right|=q^{r}$, for some $X \subset E=$ $\{1,2, \cdots, n\}$, and natural number $r$. Hence $C^{s}$ is an almost affine code over $F^{s}$, since $C$ is an almost affine code over $F$. Moreover the matroid $M_{C^{s}}=M_{C}$ since the rank functions are the same. Call the rank function $r$. Put $k=r(E)$.

Let $U \subset E$, and let $c_{Q}$ be a fixed codeword in $C^{s}$. Similarly as in [7] we define: $S_{U}(s)$ is the subset of $C^{s}$, viewed over $F^{s}$, with the same coordinates as $\boldsymbol{c}_{\boldsymbol{Q}}$ in the positions corresponding to $U$, in other words $S_{U}(s)=C^{s}\left(U, \boldsymbol{c}_{\boldsymbol{Q}}\right)$. But, since $C^{s}$ is an almost affine code we see that $\left|S_{U}(s)\right|=\left(q^{s}\right)^{k-r(U)}$. In the next definition, there is no explicit reference to the codeword $c_{\boldsymbol{Q}}$, since this is independent of the word chosen.

Definition 10. For each $j=1, \cdots, n$ let $A_{C, j}(s)$ be the number of codewords of weight $j$ in $C^{s}$.

Using the exclusion/inclusion principle we we obtain the same formula as in [7, Formula (9) p. 638]:

$$
A_{C, n}(s)=(-1)^{n} \sum_{U \subset E}(-1)^{|U|}\left(q^{s}\right)^{n^{*}(U)} .
$$

Here $n^{*}(Y)=|Y|-r^{*}(Y)$ is the nullity function associated to the dual matroid $\left(E, r^{*}\right)$ of $M_{C}$.

For each $X \subset E$ let $a_{X, C}(s)$ be the number of codewords with support exactly $X$. We then obtain in a similar way: 


\section{Lemma 1.}

$$
a_{X, C}(s)=(-1)^{|X|} \sum_{U \subset X}(-1)^{|U|}\left(q^{s}\right)^{n_{X}^{*}(U)},
$$

where $n_{X}^{*}$ is the nullity function of the dual of the rank function associated to the code $C\left(E \backslash X, c_{Q}\right)$.

A refined study, using Proposition 3, also gives

Lemma 2. For any $U \subset X$ we have: $n_{X}^{*}(U)=n^{*}(U)$.

Combining Lemmas 1 and 2 we then obtain an analogous formula as in [7, p. 638], and obtain:

This gives:

Proposition 5. For each $j=0,1, \cdots, n$ there are polynomials

$$
A_{C, j}(s)=(-1)^{j} \sum_{|X|=j} \sum_{Y \subset X}(-1)^{|Y|}\left(q^{s}\right)^{n^{*}(Y)} .
$$

counting the number over codewords of weight $j$ in $C^{s}$.

In [7, Sections 4 and 5], one shows how this matroid expression can be expressed by $\mathbb{N}_{0}$-graded Betti numbers of the Stanley-Reisner rings of the matroid $M_{C}^{*}$ and its elongations, viewed as simplicial complexes via their independence sets ([7, Theorem 5.1]). From the arguments above we now see that its concequence, [7, Corollary 5.1], formulated for linear codes in that corollary, carries over to almost affine codes, except that the matroid $M(H)$ appearing in [7, Corollary 5.1], must be replaced by the matroid dual $M_{C}^{*}$. See also [7, Proposition 4.1], which can be applied to determine the generalized Hamming weights for almost affine codes from the degrees of the polynomials $A_{j}(s)$.

We also observe:

Example 1. As one sees from Proposition 5 the formula for $A_{C, j}(s)$ is only dependent on the polynomial (in the variable $Q$ )

$$
P_{j}(Q)=(-1)^{j} \sum_{|X|=j} \sum_{Y \subset X}(-1)^{|Y|}(Q)^{n^{*}(Y)},
$$

which is defined for any (demi-)matroid, since it only uses its dual nullity function.

Let $C$ be the almost affine code in [14, Example 2]. This is a code of rank 3 over the alphabet $\mathbb{F}_{3}^{2}$ of cardinality 9 . Its length is also 9 , and its well known ([14, Example 2]) that its associated matroid $M_{C}$ is the non-Pappus matroid. In [4, p. 102] one calculated the polynomials $P_{j}(Q)$, for $j=0, \cdots, 9$ without relating them to any code, since one knew that this matroid is not linearly representable. The results, however, automatically carry over to determining the $A_{C, j}(s)$ for the non-linear almost affine code $C$, and we obtain from [4, p. 102], or from usual inclusion/exclusion methods:

$$
A_{C, 0}(s)=1,
$$




$$
\begin{gathered}
A_{C, 1}(s)=A_{C, 2}(s)=A_{C, 3}(s)=A_{C, 4}(s)=A_{C, 5}(s)=0, \\
A_{C, 6}(s)=8 q^{s}-8, \\
A_{C, 7}(s)=12 q^{s}-12, \\
A_{C, 8}(s)=3 q^{2 s}-18 q^{s}+15, \\
A_{C, 9}(s)=q^{3 s}-9 q^{2 s}+28 q^{s}-20 .
\end{gathered}
$$

$q^{3 s}=\left|C^{s}\right|$.

\section{References}

1. T. Britz, T. Johnsen, D. Mayhew, and K. Shiromoto, Wei-type duality theorems for matroids, Designs, Codes Cryptogr., vol. 62, no. 3, pp. 331-341, 2012.

2. G.F. Forney, Dimension/Length Profiles and Trellis Complexity of Linear Block Codes, IEEE Trans. Inform. Theory, vol. 40, no. 6, pp. 1741-1752, 1994.

3. O. Geil, S. Martin, R. Matsumoto, D. Ruano, Relative generalized Hamming weights of one-point algebraic geometric codes, IEEE Trans. Inform. Theory, vol. 60, no. 10 pp. 5938-49, 2014.

4. V. Huerga Represa, Towers of Betti Numbers of Matroids and Weight Distribution of Linear Codes and their Duals, Master's thesis in Pure Mathematics, University of Troms $\varnothing$ - The Arctic University of Norway, 2015. Available at http://hdl.handle.net/10037/7736.

5. R.P.M.J. Jurrius, Weight enumeration of codes from finite spaces Des. Codes Cryptogr., vol. 63, no. 3, pp. 321-330, 2012.

6. R.P.M.J. Jurrius and G.R. Pellikaan, Algebraic geometric modeling in information theory, In: Codes, arrangements and matroids. Seroes on Coding Theory and Cryptology. World Scientific Publishing, Hackensack, NJ, 2001

7. T. Johnsen, J. Roksvold and H. Verdure, Generalized weight polynomials of matroids, Discrete Math., vol. 339, no. 2, pp. 632-645, 2016.

8. T. Johnsen, H. Verdure, Hamming weights of linear codes and Betti numbers of Stanley-Reisner rings associated to matroids, Appl. Algebra Engrg. Comm. Comput., vol. 24, no. 1, pp. 73-93, 2013.

9. T. Johnsen, , H. Verdure, Generalized Hamming weights for almost affine codes, IEEE Trans. Inform. Theory, vol. 63, no. 4, 1941-1953, 2017.

10. T. Johnsen, H. Verdure, Flags of almost affine codes, arXiv:1704.02819, 2017.

11. J. Kurihara, T. Uyematsu, R. Matsumoto, Secret sharing schemes based on linear codes can be precisely characterized by the relative generalized Hamming weights, IEICE Transactions on Fundamentals of Electronics, Communication and Computer Sciences, vol. 95, no. 11, pp. 2067-75, 2012.

12. Z. Liu, W. Chen, Yuan Luo, The relative generalized Hamming weight of linear qary codes and their subcodes, Designs, Codes Cryptogr., vol. 48, no. 2, pp 111-123, 2008.

13. J.G. Oxley, Matroid theory, Oxford University Press, 1992.

14. J. Simonis and A. Ashikhmin, Almost Affine Codes, Des. Codes Cryptogr., vol. 14, no. 2, pp. 179-197, 1998.

15. Z. Zhuang, B. Dai, Y. Luo, A.J. Han Vinck, On the relative profiles of a linear code and a subcode, Designs, Codes Cryptogr., vol. 72, no. 2, pp. 219-247, 2014. 